\title{
La agenda política en las elecciones de abril de 2019 en España: programas electorales, visibilidad en Twitter y debates electorales
}

\section{The political agenda in the Spanish election of April 2019: Electoral programs, Twitter, and electoral debates}

\author{
Antón R. Castromil; Raquel Rodríguez-Díaz; Paula Garrigós
}

Cómo citar este artículo:

Castromil, Antón R.; Rodríguez-Díaz, Raquel; Garrigós, Paula (2020). “La agenda política en las elecciones de abril de 2019 en España: programas electorales, visibilidad en Twitter y debates electorales". El profesional de la información, v. 29, n. 2, e290217.

https://doi.org/10.3145/epi.2020.mar.17

Artículo recibido el 10-10-2019

Aceptación definitiva: 09-01-2020

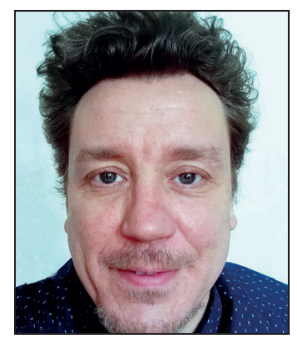

Antón R. Castromil $ه$

https://orcid.org/0000-0001-9461-6647

Universidad Complutense de Madrid

Facultad de Ciencias de la Información

Av. Complutense, 3

28040 Madrid, España

arcastromil@ccinf.ucm.es

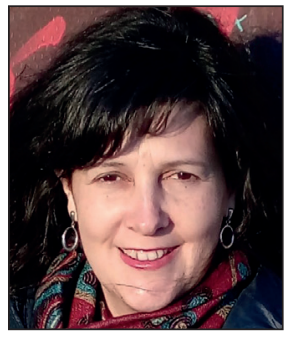

Raquel Rodríguez-Díaz

https://orcid.org/0000-0002-8097-6585

Universidad Rey Juan Carlos

Facultad de Ciencias de la Comunicación

Camino del Molino, $\mathrm{s} / \mathrm{n}$

28943 Fuenlabrada (Madrid), España

raquel.rodriguez@urjc.es

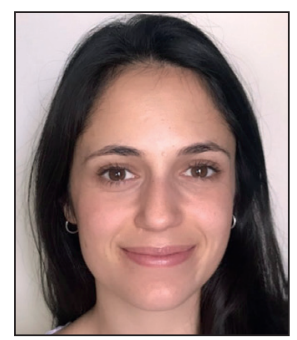

Paula Garrigós

https://orcid.org/0000-0002-8549-4147

Universidad Rey Juan Carlos

Facultad de Ciencias de la Comunicación

Camino del Molino, $\mathrm{s} / \mathrm{n}$

28943 Fuenlabrada (Madrid), España

paulagarrigos94@gmail.com

\section{Resumen}

El conjunto de temas de debate que nuestros representantes públicos consideran importantes suele denominarse agenda política. Esta agenda, por comparación con las prioridades de los medios de comunicación (agenda mediática) y las de los ciudadanos (agendas pública y personal), resulta mucho más difícil de objetivar. Este artículo ensaya una metodología para conocer la composición temática de la agenda política basada en el análisis de tres repertorios de comunicación política durante las elecciones generales españolas de abril de 2019: los programas electorales de los partidos, el uso de Twitter, y los debates electorales. Se emplea el análisis de contenido para estudiar la composición temática de los programas electorales, los debates televisivos y el uso que realizaron los candidatos en Twitter.

\section{Palabras clave}

Agendas; Agenda-setting; Agenda política; Elecciones; Programas electorales; Twitter; Medios sociales; Debates electorales; Televisión; Comunicación política; España.

Financiación

Este trabajo forma parte del proyecto de investigación DEBATV, Debates electorales televisados en España: modelos, proceso, diagnóstico y propuesta (CSO2017-83159-R), proyecto de I+D+i (Retos) financiado por el Ministerio de Ciencia, Innovación y Universidades y por la Agencia Estatal de Investigación (AEI) del Gobierno de España. 


\begin{abstract}
The series of issues for debate which our public representatives consider important is normally referred to as the "political agenda". This agenda, when compared to the priorities of the media ("media agenda") and those of the general public ("public" and "private" agendas), is much more difficult to measure. This article uses a methodology to ascertain the thematic break-down of the political agenda based on the analysis of three different scenarios of political communication during the Spanish general elections held in April 2019: the electoral programs of the parties, the use of Twitter and electoral debates. This contribution uses the methodology of content analysis to study the agenda of the main issues in the electoral programs and televised debates in addition to how politicians used the social network Twitter to deal with the agenda issues.
\end{abstract}

\title{
Keywords
}

Agenda; Agenda-setting; Political agenda; General election; Electoral programs; Twitter; Social media; Electoral debates; Television; Political communication; Spain.

\section{Introducción}

Las elecciones generales celebradas en España en abril de 2019 ofrecen la oportunidad de ensayar una metodología para conocer la composición temática de la denominada agenda política. Se trata de un período electoral especialmente interesante para el estudio de la tematización de una campaña ya que, tal y como se examina en detalle en las páginas que siguen, en la legislatura 2015-2019 conviven dos presidentes del gobierno (Mariano Rajoy, 2016-2018 y Pedro Sánchez, 2018-2019) y dos ejes temáticos dominantes muy distintos entre sí. Por un lado, la denominada "regeneración democrática" (con temas de debate como la corrupción o "los políticos") y otro de corte identitario (unidad de España o independencia de Cataluña).

Este contexto político-social tan complejo ofrece una buena ocasión para poner a prueba nuestra propuesta de observación de la agenda política. En este trabajo tenemos en cuenta los programas electorales, la visibilidad temática de las cuentas de partidos y candidatos en Twitter, y la actuación de esos mismos candidatos en los dos debates electorales celebrados en las elecciones de abril de 2019.

La investigación describe primero el marco teórico que se adopta, la teoría de agenda-setting, y su reconocimiento de la multiplicidad de actores que interactúan en todo sistema democrático: el mundo político (agenda política), el periodístico (agenda mediática) y la ciudadanía (agenda pública y personal). En un segundo momento se explican las dificultades para conocer la agenda política y nuestra propuesta de medición basada en el examen de programas, Twitter y debates. Por último, acudimos a los datos de agenda política procedentes de estas tres fuentes referidos a la legislatura 20152019 y a la campaña electoral llevada a cabo en España a lo largo del mes de abril de 2019.

\section{Temas de una campaña electoral: políticos, medios y ciudadanos}

Las campañas electorales son momentos comunicativos especialmente intensos. En ellas se produce una interconexión constante entre los denominados tres eslabones de la comunicación política (Mazzoleni, 2010, p. 27):

- sistema político;

- medios de comunicación;

- ciudadanía.

La presente investigación permite conocer un poco más de cerca la instrumentalización de uno de estos tres elementos, el correspondiente a la actividad político-representativa. Tal indagación se lleva a cabo utilizando un enfoque muy concreto -la teoría de agenda-setting- por considerarla una perspectiva que ofrece una capacidad analítica adecuada al reto que nos hemos puesto: descifrar la agenda política en las primeras elecciones españolas de 2019.

El enfoque propuesto en su día por McCombs y Shaw (1972) aporta una unidad de análisis que consideramos fundamental a la hora de enfrentarnos al estudio de la actividad política en una campaña electoral. Nos referimos a los temas de debate público que los representantes irán tratando a lo largo y ancho del período electoral. Investigaciones nacionales e internacionales han empleado diferentes metodologías que han abordado estos aspectos (Chavero-Ramírez et al., 2013; Kim; Kim; Zhou, 2017; McCombs; Shaw; Weaver, 2014).

Partidos y candidatos estarán interesados en que la campaña gire de forma preferente sobre unos temas de debate público y no otros. Que la agenda temática de la campaña se parezca mucho a su propia agenda política. Esta es una de las principales batallas que tiene lugar en cualquier elección y constituye uno de los objetivos prioritarios de la denominada comunicación política (Castromil, 2017, p. 97).

Esta intención de tematización de una campaña en un sentido particular -sobre inmigración o sobre corrupción, por ejemplo- a menudo colisiona de frente con los intereses de otros muchos actores que conviven en el mismo ecosistema. De alguna forma, la agenda política se ve modificada
La agenda política ha sido mucho menos estudiada que las dos agendas tradicionales en teoría de agenda-setting: la agenda mediática y las agendas ciudadanas 
con esta interacción con los otros. Vivimos en sociedad y una campaña electoral no es sino un conjunto de relaciones entre actores de distinta índole con intereses diferentes (Miralles, 2011).

En primer lugar se sitúan los demás partidos y candidatos que en muchas ocasiones tendrán intenciones propuestas de temas de agenda diferentes y en ocasiones opuestas. Así entendemos aquí la lucha partidista como una pugna por destacar unos temas por encima de otros, y por resistir frente a las estrategias de tematización de los demás actores. En segundo lugar hay que hablar de los medios de comunicación que mediante la denominada agenda mediática (McCombs, 2006 , p. 24) aspiran a influenciar a los otros dos eslabones de la comunicación política a los que se refería Mazzoleni (2010): a los ciudadanos (agenda pública) y a los políticos (agenda política).

Es decir, parte del contenido temático final de lo que podemos denominar agenda política procede no sólo de las preferencias e intereses partidistas de los propios actores políticos. Influyen también los otros partidos, los medios de comunicación y los ciudadanos. Nuestra propuesta de reconstrucción de la agenda política tiene muy en cuenta este juego de influencias múltiples.

Una campaña electoral, en definitiva, puede ser entendida como una continua negociación en torno a una serie de temas procedentes de los más diversos lugares/actores (políticos, medios y ciudadanos) y de la interconexión entre los temas de sus agendas (Carballo; López-Escobar; McCombs, 2018; Navarro-Sorolla; Rodríguez-Díaz, 2017; Restrepo-Echavarría; Rodríguez-Díaz; Castromil, 2018).

\subsection{Agenda política: una agenda difícil de objetivar}

Los estudios de agenda-setting suelen tener muy presente la existencia de esta pluralidad de agendas. De este modo, el análisis electoral adquiere una riqueza multiplicada. La agenda clásica en agenda-setting no es sino un estudio de correlación entre la agenda de los medios o agenda mediática y la agenda pública. De esta agenda parten los principales postulados de la teoría:

- la necesidad de orientación de los ciudadanos (McCombs, 2006, p. 110) en un mundo marcado por la distancia entre temas y experiencia (Lippmann, 2011);

- la denominada hipótesis de la transferencia de importancia de temas desde la agenda de los medios hacia las agendas ciudadanas (McCombs; Shaw, 1972, p. 177).

De esta forma, los estudios clásicos de agenda-setting se centran en los temas que ofrecen los medios a sus públicos mediante el uso de la técnica del análisis de contenido (Sánchez-Aranda, 2005; Guo; McCombs, 2015). El investigador lleva a cabo un seguimiento de determinados medios de comunicación durante un período para comprobar la composición de su cobertura.

Las agendas ciudadanas (pública y personal) resultan más accesibles al menos en países como España, donde el Centro de Investigaciones Sociológicas (CIS), en sus barómetros mensuales, se encarga de ofrecer los datos correspondientes.

Sin embargo, la objetivación de la agenda política resulta mucho más complicada. ¿A dónde debemos acudir para conocer su composición exacta? No existe una respuesta tan directa como para el caso de la agenda mediática (análisis de contenido de medios) y sobre todo, las agendas ciudadanas (CIS). En esta investigación hacemos una propuesta, a la fuerza parcial, para arrojar algo de luz sobre la agenda política. Estamos seguros de que los temas de debate presentados aquí forman parte de esta agenda. El problema reside en que "no están todos los temas que son", como suele decirse. Siempre quedará algún tema en el tintero. Cuando se trata con la agenda política, debemos acostumbrarnos a trabajar con cierto grado de incertidumbre.

Los temas contenidos en esta agenda política se pueden conocer acudiendo a lugares tan distintos como el programa electoral, la actividad legislativa, los mítines y otros actos de campaña, las coberturas de los medios, la influencia de grupos y ciudadanos y la interacción con otros partidos y candidatos. ¿Cuál de estos repertorios elegir? ¿Debemos combinar varios? ¿Cuáles?

\subsection{Propuesta de medición de la agenda política}

Siendo conscientes de las limitaciones inherentes a toda sistematización de la agenda política, en nuestra investigación la conocemos, al menos en parte, a través de los programas electorales, la visibilidad temática de partidos y candidatos en la red de microblogging Twitter y los debates electorales celebrados durante la campaña.

Estos tres lugares ofrecen además una doble ventaja. Por un lado, los programas electorales y Twitter podrían considerarse formas "puras" de objetivar la agenda política. Es decir, en ambos lugares podemos obtener las preferencias temáticas de los actores políticos en liza en una campaña sin que intervenga ningún otro factor. Compárese por ejemplo con la comunicación mediatizada de un repertorio tan clásico como una rueda de prensa. De lo que el representante de un partido expresa en tal contacto con los medios a lo que termina por ser publicado puede llegar a mediar un abismo. De este modo la comunicación política "pura" tiene la ventaja de dejar a un lado cuestiones tan complejas como:

- las implicaciones de la mediatización periodística (Ortega, 2011, p. 35; Strömbäck, 2008);

- el fenómeno de la polarización (Castromil, 2017, p. 198);

- el paralelismo político-mediático (Hallin; Mancini, 2004, p. 89). 
Por otro lado, nuestra propuesta incluye dos formas distintas dentro de lo que hemos convenido en denominar comunicación "pura": programas electorales y Twitter. Teniendo en cuenta los programas aspiramos a captar los temas clásicos que los partidos utilizan en su relación con la sociedad. Aquí la selección temática se relaciona con cuestiones como la ideología (Snow, 2004), y los denominados cleavages o fracturas sociales (Lipset; Rokkan, 2001) sobre las cuales los partidos operan. Los principales suelen ser la clase social, la religión y la pertenencia nacional.

Resulta bastante probable que los partidos a través de sus programas señalen, por así decirlo, los temas "de toda la vida" de las tradiciones político-ideológicas a las que pertenecen. Cuando McCombs (2006) sostenía que algunos temas tienen dueño se refería justamente a esto. Así, la izquierda suele verse como una opción más fuerte en cuestiones como sanidad, política social o educación; y la derecha en economía, inmigración y seguridad ciudadana. La cultura política (Benedicto, 1995) enlaza aquí con los procesos de tematización.

De este modo un análisis de contenido riguroso de los programas electorales ofrece algo de certidumbre sobre este tipo de cuestiones. Sin embargo, el estudio de los mecanismos "puros" de tematización creemos que debe complementarse con otras fuentes más dinámicas. De ahí que hayamos recurrido a Twitter, una plataforma online que si por algo puede caracterizarse es por su capacidad de acercamiento a los acontecimientos que suceden en cada momento. Entendemos Twitter como una actualización y aplicación constante de las grandes líneas ideológico-programáticas a los acontecimientos de la actualidad, como reflejan varios estudios (López-López; Vásquez-González, 2018; Ruiz-del-Olmo; Bustos-Díaz, 2017; Vu; Guo; McCombs, 2014).

Desde Twitter los partidos y candidatos que se presentaron a las primeras elecciones de 2019 reaccionaron a lo que sucedía, seleccionando unos temas por encima de otros y haciendo compatibles las nuevas problemáticas con sus estrategias de tematización de la campaña.

Si los programas electorales informaban de los temas más clásicos o, si se quiere, estáticos de la agenda política; es probable que dando cabida a Twitter podamos conocer también las preferencias temáticas más instantáneas y sobre todo su relación con el universo significativo maestro (Snow; Benford, 2006) de ese mismo partido político.

Pero el acceso a la comunicación "pura”, tanto en su versión más estática (programas electorales) como dinámica (Twitter), parece una condición necesaria, aunque no suficiente para conocer con un mínimo de exactitud la agenda política. Nos faltaría atender a las interacciones con otros actores de la campaña electoral. Porque parte de lo que partidos y candidatos son, parecen y representan se debe a su relación con otros participantes de la vida social. Como hemos comentado más arriba nos referimos, sobre todo, a ciudadanos (agenda pública y personal) y medios de comunicación (agenda mediática).

Los debates electorales y los bloques sobre economía, política social, política territorial o asuntos exteriores, por poner sólo unos cuantos ejemplos, fuerzan a partidos y candidatos a posicionarse en temas que podrían o no estar en sus respectivas agendas políticas. O haberse tratado muy de refilón.

La comunicación que surge de estos debates sigue siendo un tipo de comunicación política "pura" en el sentido de que no se encuentra mediatizada en su contenido por otros actores. Sin embargo, el espectro temático se amplía debido a la principal característica de todo debate electoral (García-Marín; Calatrava; Luengo, 2018; Wagner, 2016): la interacción con otros partidos y candidatos.

Como veremos en el apartado de resultados, tales bloques temáticos que debieron tratarse proceden de las preocupaciones ciudadanas (temas de agenda pública) y de las prioridades de los periodistas (agenda mediática). De este modo damos entrada a ciudadanos y periodistas en nuestra propuesta de codificación de la agenda pública de cara a las elecciones de 2019.

\section{De la regeneración democrática a la unidad de España}

La tematización de las agendas se relaciona de manera estrecha con la actualidad. Es cierto que en agenda-setting suele considerarse necesario el transcurso de un cierto tiempo para que los temas entren en las prioridades de partidos, medios y ciudadanos (McCombs, 2006, p. 92; Rodríguez-Díaz, 2004, p. 34). McCombs cifra en entre "cuatro y ocho semanas" (McCombs, 2006, p. 95) el período necesario para que un acontecimiento entre en la agenda de los medios.

Sea este lapso de tiempo el necesario u otro más corto o extenso, lo que parece imprescindible es acudir al contexto previo de unas elecciones si de lo que se trata es de estudiar la composición temática de la agenda de nuestros representantes. Entendemos aquí que lo político surge en un contexto y que ese contexto explica al menos parte de las preferencias temáticas de los actores en disputa en una campaña.

Desde que en 2015 el sistema de partidos español abandonase la lógica bipartidista PP-PSOE imperante desde 1982 (Castromil; Rodríguez-Díaz, 2019), la vida política se ha movido a una gran velocidad. La legislatura 2016-2019 ofrece una buena muestra de ello. Acontecimientos y temas de debate transcurren con gran rapidez. 
En primer lugar, hay que recordar que las de abril de 2019 son unas elecciones anticipadas, ya que la legislatura bien podría haberse extendido hasta mediados de 2020. El presidente, el socialista Pedro Sánchez, decide adelantar las elecciones ante la imposibilidad de aprobar los presupuestos para ese año 2019.

\section{La legislatura 2016-2019 supone una evolución desde temas de regeneración democrática y corrupción hacia otros como la independencia de Cataluña}

Sin embargo, la legislatura había arrancado de forma bien diferente. Con Mariano Rajoy (Partido Popular), ocupando el poder (octubre 2016-junio 2018) gracias a la abstención de parte del grupo parlamentario del PSOE. Aunque terminarían siendo también los socialistas los que lo expulsarían del poder mediante una moción de censura en junio de 2018.

Un importante tema de debate estaba detrás de la primera moción de censura exitosa de la historia democrática reciente: la corrupción del denominado "caso Gürtel"1. Esta circunstancia aglutinaría una insólita coalición anti $P P(P S O E, U P$, ERC, PDCat, PNV, Compromís, Bildu y Nueva Canarias) que auparía a Pedro Sánchez a la presidencia del Gobierno. El PP pasaba así a la oposición y Pablo Casado se hacía con las riendas del partido.

Sin embargo la corrupción, la regeneración democrática o incluso "los políticos", tal y como suele codificarlo el $C I S^{2}$, irían poco a poco perdiendo centralidad en la legislatura. Dos acontecimientos importantes explican al menos parte del declive de los temas de debate de "regeneración" y la entrada de la "unidad de España".

En primer lugar, hay que hablar de la activación del movimiento independentista en Cataluña (Guillén-Bravo; Rodríguez-Díaz, 2017). En la legislatura se celebra un referéndum muy polémico ${ }^{3}$ sobre su independencia, se amaga con una declaración unilateral de independencia (DUI) y se aplica, por parte del Gobierno de Mariano Rajoy, el artículo 155 de la Constitución 4 . Todos estos acontecimientos hacen de la "independencia de Cataluña" o de la "unidad de España" un tema de atención máxima por parte de los medios de comunicación y el sistema político español. Y por supuesto, también de los ciudadanos, tal y como muestran los datos de agenda pública que examinaremos en el epígrafe 5.

En segundo lugar, la sustitución de Rajoy por Sánchez tras la moción de censura, aunque se debió a cuestiones relacionadas con el tema de debate "corrupción", puede entenderse también como la introducción de la "unidad de España" en el tablero de juego nacional, no sólo en el catalán. Debemos tener en cuenta que gran parte del éxito de Pedro Sánchez se debe al apoyo de partidos regionalistas, nacionalistas o independentistas. Esta circunstancia no pasó inadvertida y terminaría por colocar a la "unidad de España" en un lugar preferente del debate público.

De alguna forma, la sustitución de la "regeneración democrática" por cuestiones de identidad nacional (España o Cataluña) no fue algo neutro para los partidos. Como hemos dicho ya, algunos temas parecen tener dueño (McCombs, 2006). Teniendo en cuenta esta circunstancia resulta más sencillo entender el éxito de partidos como Ciudadanos, que casi supera al PP como principal partido de la derecha ${ }^{5}$ o la eclosión de Vox. Este partido ultraderechista consiguió en abril de 201924 diputados en estas elecciones (10,26\% de los votos). En la izquierda, el PSOE no resultó demasiado dañado por este tema de debate -aunque, como veremos más adelante, tampoco lo sitúa entre sus favoritos- mientras que Podemos sí sufrió por este viraje desde cuestiones sociales (regeneración, clase política, la casta...) hacia cuestiones de identidad española.

\section{Metodología}

Nuestra propuesta de medición de la agenda política incluye el análisis de los programas electorales de los principales partidos que han concurrido a las elecciones generales españolas de abril de 2019. Pero también su visibilidad temática en Twitter y sus intervenciones en los debates electorales televisados celebrados los días 22 (RTVE) y 23 de abril (Atresmedia). Examinamos a continuación cada uno de estos tres elementos.

\subsection{Programas electorales}

Hemos accedido a los principales contenidos de los programas electorales de las cinco principales fuerzas políticas que se presentaron a las elecciones generales de abril de 2019 mediante la herramienta de comparación de programas del diario El país ${ }^{6}$. En la aplicación es posible comparar los programas del PSOE, PP, C's, UP y Vox en cuestiones como educación, políticas de igualdad, inmigración, transición ecológica, sanidad, crisis territorial, empleo, impuestos, política social, España abandonada (vaciada) y pensiones.

El análisis incluye el número de propuestas identificadas para cada tema y el total de propuestas. Este último dato permite conocer la mayor o menor visibilidad de cada eje temático, pudiendo así hacernos una idea de la tematización preferida por cada opción partidista.

\subsection{Twitter}

Se ha llevado a cabo un análisis de interactuación de cuentas de Twitter desde el inicio de la campaña el 12 de abril de 2019 hasta su finalización el 25 de ese mismo mes. En total se han seguido diez cuentas que corresponden tanto a la cuenta oficial del partido como a la de su candidato a la presidencia del Gobierno. Es decir, se optó por realizar un seguimiento dual partido/líder, lo que permite conocer el grado de coherencia, refuerzo y estrategia conjunta entre la estructura partidista y el liderazgo personal de cada candidato. 
Las cuentas analizadas fueron:

- @PSOE, @sanchezcastejon

- @populares, @pablocasado_

- @CiudadanosCs, @Albert_Rivera

- @ahorapodemos, @Pablo_Iglesias_

- @VOX_es; @Santi_abascal.

Consideramos interacción los tweets o retweets que proceden de cada cuenta. Entendemos tweet como un contenido que se publica en una cuenta dentro de la red social que es elaborado por el propio usuario o su equipo. Un retweet será todo aquel contenido elaborado por otro usuario o conjunto de usuarios que alguna de nuestras cuentas objeto de estudio decide compartir con su comunidad de seguidores.

Para el análisis de estas diez cuentas de Twitter hemos utilizado Twitonomy. Esta aplicación, en su versión de pago, permite extraer todas las publicaciones de una cuenta de Twitter, lo que incluye tanto tweets como retweets. El tratamiento posterior de datos permitió filtrar y obtener sólo aquel material referido a los principales temas de debate de las elecciones generales de abril de 2019.

\subsection{Debates electorales televisivos}

En las elecciones generales españolas de abril de 2019 se celebraron por primera vez en la historia democrática reciente dos debates en días consecutivos. El primero tuvo lugar en Televisión Española (TVE) y fue moderado por el periodista Xabier Fortes. El segundo corrió a cargo del grupo Atresmedia y contó con un formato algo más informal y abierto. Los moderadores fueron Ana Pastor y Vicente Vallés.

Una institución clave en toda campaña electoral en España es la Junta Electoral Central, ya que dentro de los términos que regula la Ley Orgánica de Régimen Electoral General (Loreg), es el organismo encargado de velar por la proporcionalidad y el juego limpio en campaña, lo cual incluye por supuesto los debates electorales. En abril de 2019 este organismo tuvo un papel muy importante, ya que impidió que el formato evolucionase desde el modelo "a cuatro" (PSOE, $P P, U P$ y C's) puesto en marcha en 2015 y 2016 (Castromil; Rodríguez-Díaz, 2019, p. 34) hacia otro "a cinco", que hubiera dado cabida también al partido emergente Vox ${ }^{7}$.

Para analizar los temas que se trataron en los debates electorales se procedió a su visionado íntegro y a la contabilización del tiempo (en segundos) dedicado por cada uno de los candidatos a cada tema de debate. De esta forma, pudimos observar la visibilidad que cada candidato dio a cada cuestión de debate público que se proponía dentro de las reglas y tiempo que se marcaban desde la organización del debate.

\section{Resultados}

Antes de comenzar con el estudio de los principales componentes temáticos de la agenda política a través del análisis de programas electorales, Twitter y los debates, vamos a repasar la composición de la agenda pública que ofrece el Centro de Investigaciones Sociológicas $(C / S)^{8}$. De esta forma, es posible comparar la agenda política que vamos a ir ofreciendo aquí con las principales preocupaciones de los españoles a lo lago de la legislatura 2016-2019.

Como suele ser habitual, las cuestiones económicas protagonizan esta agenda ciudadana, sobre todo el paro (valor promedio: $\left.66,9 \%{ }^{9}\right)$, los problemas de índole económica $(22,4 \%$ y otras cuestiones relacionadas con los servicios que presta el estado del bienestar al ciudadano como la sanidad, (11,3\%), los problemas sociales $(9,2 \%)$ o la educación ( $9 \%)$. Junto a estos elementos, la agenda pública de la legislatura da cabida también a temas de cariz más político, que habían entrado en ella a partir de la crisis económica iniciada en 2008. Son sobre todo la corrupción (valor promedio: 36,1\%) y los políticos (24,9\%).

Estos siete temas constituyen el eje temático principal de las preocupaciones ciudadanas a lo largo de la legislatura 2016-2019. Sin embargo, como parece lógico, algunas otras cuestiones han entrado y salido de los primeros puestos de esta agenda dependiendo de la actualidad del momento. Nos referimos a temas como los ya tratados sucesos en Cataluña (referéndum, declaración de independencia...), el de-

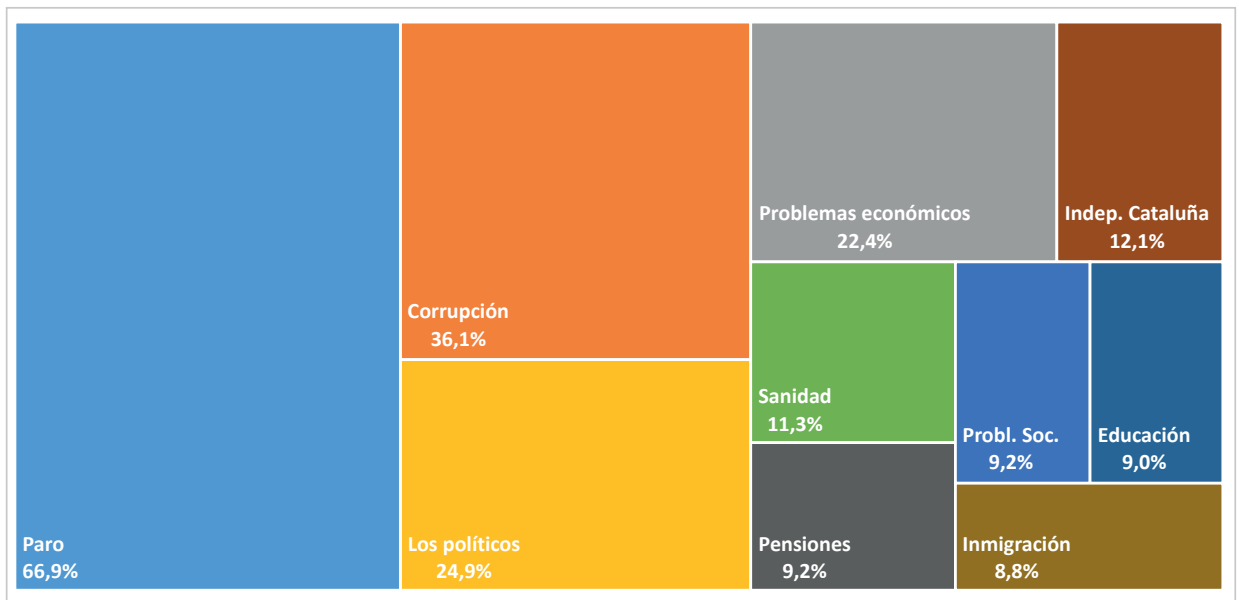

Gráfico 1. Promedio de las principales preocupaciones ciudadanas (agenda pública) 2016-2019. Fuente: Barómetros CIS (julio 2016-febrero 2019) 
bate sobre la sostenibilidad de las pensiones o la política migratoria ${ }^{10}$.

La independencia de Cataluña, tal y como la codifica el $C / S$, entra de forma abrupta en la agenda pública de los españoles en octubre de 2017 (29\%) -con la celebración del referéndum de independencia del 1-Oy se mantendrá en lugares de relativa importancia hasta mayo de 2018 , cuando inicia un paulatino declive, con picos importantes en septiembre $(13 \%)$ y octubre $(12,2 \%)$ de ese mismo año. Algo parecido sucede con las pensiones: los meses de mayor importancia en la agenda pública se encuentran entre marzo $(15,5 \%)$ y julio $(9,3 \%)$ de $2018^{11}$. Por último, la inmigración adquiere también una importancia relativa en la legislatura, pero sólo a partir del verano de 2018 ( $15,6 \%$ en septiembre) y se mantiene en valores cercanos al $10 \%$ hasta el umbral mismo de las elecciones.

Si comparamos los valores de los diez temas de debate del gráfico 1 a lo largo de la legislatura podemos comprobar (gráfico 2) qué temas han perdido importancia y cuáles la han ganado conforme se acercaba el momento de acudir a las urnas, esto es, abril de 2019. De esta manera nos haremos una idea más fidedigna de la solidez y actualidad de las preocupaciones de la agenda pública a la puerta misma de las elecciones.

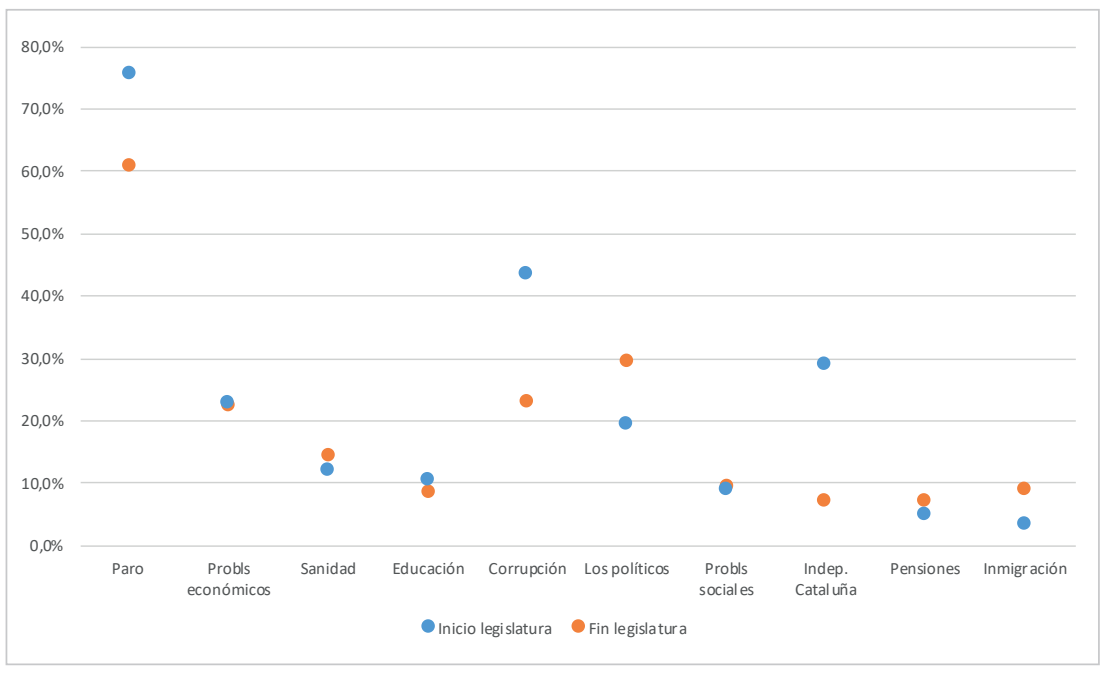

Gráfico 2. Aumento-descenso de importancia de temas de agenda pública (2016-2019). Fuente: Barómetros CIS (julio 2016-febrero 2019)

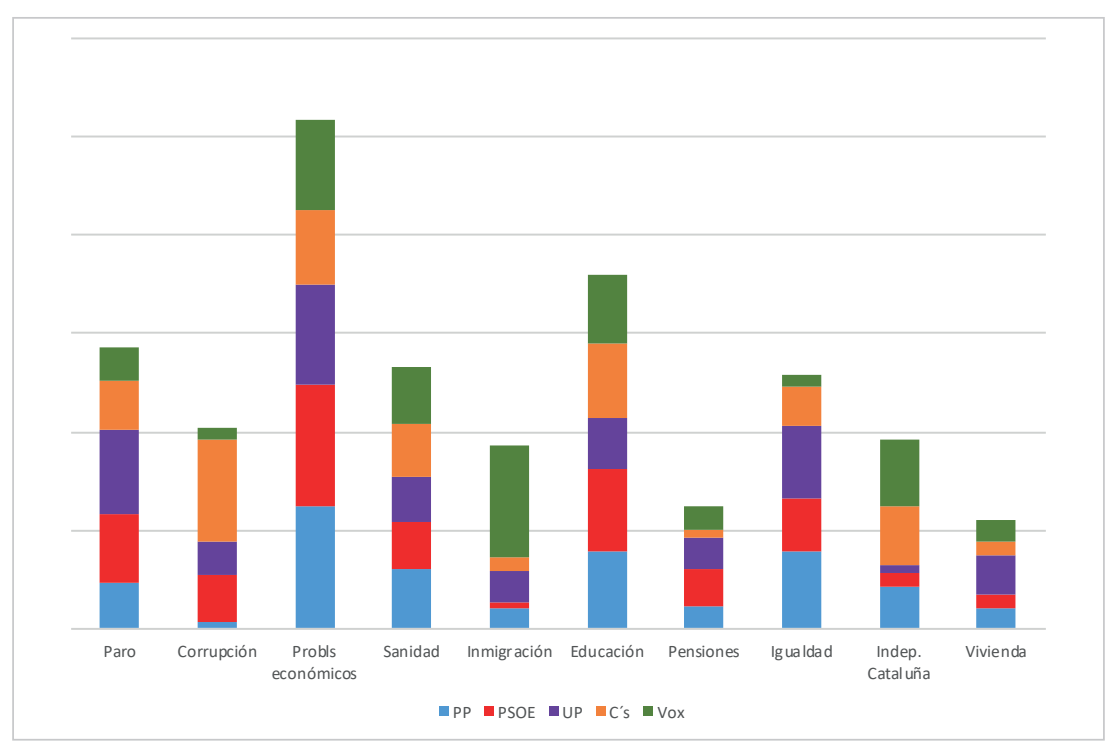

Gráfico 3. Composición temática de programas electorales (abril 2019).

Fuente: Aplicación de comparación de programas electorales del diario El país

El descenso de importancia más pronunciado se observa en los temas de debate corrupción, paro e independencia de Cataluña. Hay que tener en cuenta que el paro, a pesar de esta bajada, se mantiene como el principal problema de la agenda pública durante toda la legislatura. En referencia al Procés catalán, debemos mencionar que se trata de uno de los temas más volátiles de la agenda pública del período, al ir sujeto a oscilaciones relacionadas con acontecimientos como el referéndum del 1 de octubre de 2017 o la conmemoración de su primer aniversario un año después.

En cuanto a los temas relacionados con la regeneración democrática, la corrupción se ve sobrepasada por los políticos como segunda preocupación más importante de esta agenda ciudadana. Estos dos temas ocupan los nada desdeñables rangos 2 y 3 de la agenda pública en los momentos previos a la celebración de las elecciones. El resto de los temas permanecen más o menos constantes a lo largo de toda la legislatura.

\subsection{Programas electorales}

Las preferencias temáticas de los partidos aparecen reflejadas de manera preferente en sus respectivos programas electorales. El gráfico 3 muestra los porcentajes de cada tema de debate tomando como base el total de propuestas de los partidos. El gráfico incluye cinco de los siete temas ya tratados en la agenda pública (gráficos 1 y 2). Los políticos quedan fuera de los programas de los cinco partidos objeto de estudio y el tema de debate "políticas sociales" se desagrega en los programas en algunas políticas concretas como igualdad o vivienda.

Inmigración e independencia de Cataluña son cuestiones que hemos catalogado en la agenda pública como emergentes, que surgen al calor de ciertos acontecimientos y debates sociales y que, más tarde, tienden a bajar posiciones en la agenda pública. La independencia de Cataluña pasa de un 29\% de importancia en octubre de 2017 a sólo un 6,3\% nueve meses después (barómetro de julio de 2018). 
Sin embargo, para estas cuestiones los partidos reservan un lugar importante en sus propuestas programáticas. Comenzando por las del PSOE y el PP hay que decir que sus ejes temáticos se parecen bastante. Ambos partidos ponen especial énfasis en la economía, la educación y la igualdad. Los socialistas priman quizá algo más el empleo y los populares la cuestión de la independencia de Cataluña.

Teniendo en cuenta que tan relevante es la tematización de la campaña como aquellos otros temas que apenas aparecen en ella, por suponer una amenaza para las líneas estratégicas de la campaña, cabe destacar que el PP es el partido que pasa en mayor medida de puntillas sobre cuestiones relacionadas con la corrupción. La ya comentada moción de censura exitosa de junio de 2018 en torno a la corrupción del caso "Gürtel" parece un elemento a evitar a toda costa.

Del lado de los nuevos partidos hay que destacar la clara apuesta de Unidas Podemos por las cuestiones económicas (economía y paro), a las que se les une uno de los principales pilares del denominado estado del bienestar, la educación. Y del mismo modo que hacía el PP con la corrupción, UP destaca por ser el partido que menos trata de la cuestión catalana. Ya se sabe la dificultad que encuentran los partidos políticos situados a la izquierda del PSOE para lidiar con una idea de España que resulte convincente para sus electores. Nuestros datos indican que se trata de un tema de debate mucho más atractivo para la derecha.

El tema más importante en el programa de Ciudadanos parece ser la corrupción y el fraude, seguido de la economía y la educación. Por otro lado, cabe destacar que el partido de Albert Rivera es, junto con Vox, la formación política con más contenido programático referido a la independencia de Cataluña.

Por último, cabe destacar que la principal novedad de estas elecciones -el partido ultra Vox- muestra un eje temático muy distinto al resto de fuerzas políticas, al hacer de la inmigración el principal tema de su programa electoral, seguido de la economía, la independencia de Cataluña y la educación. El partido de Santiago Abascal apenas reserva sitio en su programa para tratar cuestiones de igualdad.

\subsection{Visibilidad en Twitter}

El gráfico 4 nos informa de al menos dos cuestiones preliminares. Por un lado, podemos observar que el partido que más ha utilizado Twitter durante la campaña de abril de 2019 fue Ciudadanos, seguido del PSOE.

Por otro lado, el gráfico deja bien clara también la preferencia de tematización en esta plataforma. En todos los partidos políticos objeto de estudio la cuenta institucional tuvo mucha más presencia que la del candidato. Esto resulta algo muy pronunciado en Ciudadanos. De entre las cuentas de los cinco aspirantes a presidente del Gobierno, las de mayor actividad fueron por este orden las de Pedro Sánchez del $P S O E$, y la de Santiago Abascal de Vox.

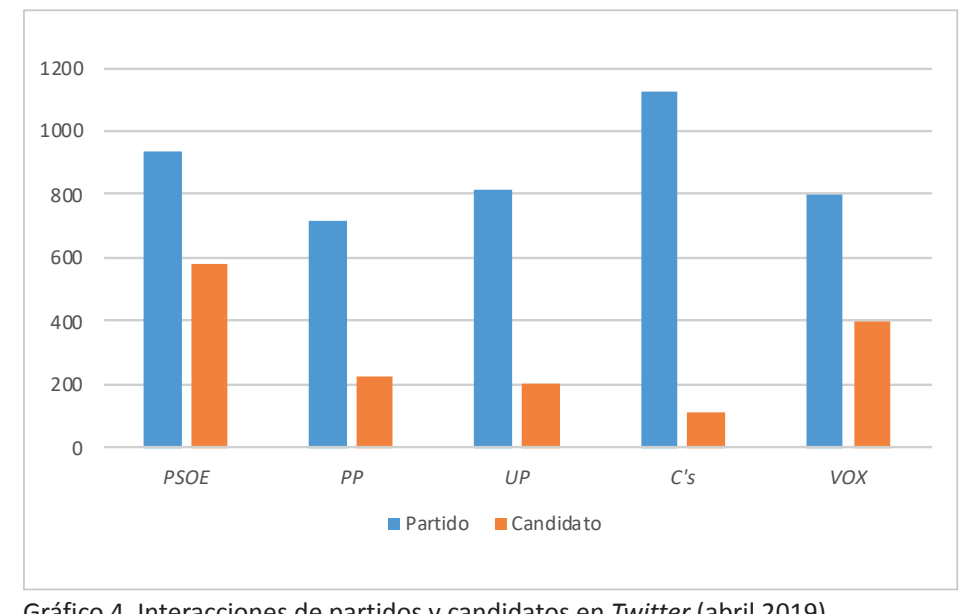

Gráfico 4. Interacciones de partidos y candidatos en Twitter (abril 2019)

Por este motivo, hemos considerado sólo las cuentas oficiales de los partidos (gráfico 1 de los Anexos) y, dentro de éstas, se dará cabida en exclusiva a las interacciones sobre los temas ya analizados en la agenda pública de los ciudadanos (gráfico 1) y en los programas electorales de esos mismos partidos (gráfico 3).

En Twitter los partidos publicaron un buen número de interacciones no relacionadas de forma directa con los temas de agenda pública expuestos con anterioridad. Durante la campaña se han identificado otros temas con mucha relevancia en las interacciones, como por ejemplo referencias a sucesos de actualidad, actividades de campaña, estado de ánimo de miembros del partido o temas que hemos considerado menores (crisis de Venezuela, sostenibilidad, defensa de los animales...)

De entre los temas que hemos analizado, por coincidir con los ya analizados en programas electorales y en la agenda pública de los ciudadanos, destacan los políticos. Son los temas más importantes en casi todos los partidos y revelan de forma muy clara el tipo de medio en el que nos estamos moviendo. A diferencia de la soledad de los programas electorales que acabamos de analizar, Twitter permite interacciones más frescas, directas y mordaces para con los rivales políticos.

Tanto el PSOE como el PP basan el grueso de sus interacciones en torno a los políticos, el paro y la independencia de Cataluña. En la cuenta del Partido Popular destaca el poco peso de la corrupción -en la línea de lo ya comentado en los programas electorales- frente a la relativa importancia que tiene entre los socialistas (4a posición).

El principal eje temático de las interacciones que parten de la cuenta oficial de Ciudadanos tiene que ver con la independencia de Cataluña, seguido de cerca de los problemas de índole económica y los políticos. Por su parte, Unidas Podemos se centra más en los políticos y cuestiones económicas (paro, problemas económicos, vivienda o pensiones). Tal y como sucedía en su programa electoral, la cuestión de la independencia de Cataluña ocupa una posición menor. 
Por último, Vox lleva a cabo una campaña en Twitter muy volcada en los políticos (contenido crítico hacia la política "tradicional"). Le siguen a cierta distancia la independencia de Cataluña y la economía. La inmigración, al contrario que el resto de las formaciones políticas en pugna en la campaña, ocupa un lugar destacado en la acción de Vox en Twitter.

\subsection{Debates electorales}

Los dos debates electorales celebrados en campaña (22 de abril, TVE; 23 de abril, Atresmedia) forzaron a los candidatos a posicionarse en todos y cada uno de los bloques temáticos propuestos. En el debate celebrado en TVE se establecieron los siguientes:

1) Política económica, fiscal y empleo.

2) Política social, estado del bienestar, pensiones e igualdad.

3) Política territorial.

4) Regeneración democrática y pactos postelectorales.

En el debate de Atresmedia del día siguiente se optó por sólo tres:

1) Presentación de los programas electorales.

2) Modelo territorial / Cataluña.

3) Pactos.

Este encorsetamiento, aunque se trate de conjuntos de temas muy amplios, constituye la diferencia principal entre este repertorio de comunicación política y los programas electorales y la visibilidad en Twitter ya analizados. Mientras en los programas y Twitter los partidos y candidatos pudieron llevar a cabo una tematización más o menos libre de condicionamientos (más en los programas que en el microblogging, como hemos visto), no sucede así a la hora de debatir en televisión.

En la medida en que los equipos de campaña pactan la estructura del debate (Gallego-Reguera; Martínez-Martínez, 2019), los candidatos están obligados a respetarla. Es decir, a dedicar un tiempo a unos temas de debate que quizá no dedicarían bajo otras condiciones. Por este motivo la comparación entre los datos que hemos obtenido respecto al énfasis de los candidatos sobre unos temas u otros (gráfico 5) con los de temas de los programas electorales y de Twitter tiene más sentido que nunca.

El debate celebrado el 22 de abril en TVE contó con una estructura algo más rígida y el de Atresmedia del día siguiente introdujo un poco más de dinamismo, ya que las preguntas de los periodistas, así como las interrupciones e interlocuciones estuvieron permitidas. Además los tiempos por bloques temáticos fueron algo más laxos.

Otra de las diferencias entre los dos debates tiene que ver con la circunstancia de que en el debate de TVE se presentaba un bloque temático y, a renglón seguido, los participantes ofrecían su posicionamiento sobre la cuestión. Por el contrario, en el debate de Atresmedia eran los periodistas los que planteaban a los líderes preguntas de actualidad a las que debían dar respuesta. Todo ello además de, por supuesto, fijar sus posturas en cada uno de los bloques del debate. El tema más tratado (gráfico 5) en ambos debates fue con diferencia la independencia de Cataluña. A esta cuestión le siguieron la economía, los políticos y el paro.

Si lo que tenemos en cuenta ahora es la distribución de estos temas por candidato a la presidencia del Gobierno (gráfico 6) vemos que los partidos que más han incidido en la cuestión catalana fueron Ciudadanos y Unidas Podemos. Como hemos visto al analizar los datos de programas electorales y sobre todo Twitter, se trata de un tema muy importante para la formación de Albert Rivera y que supone una especie de marca diferencial con el $P P$ en su lucha por el voto descontento por motivos de identidad.

Sorprende aquí la segunda posición en el tratamiento de este tema (independencia de Cataluña): Unidas Podemos. Recordemos que se

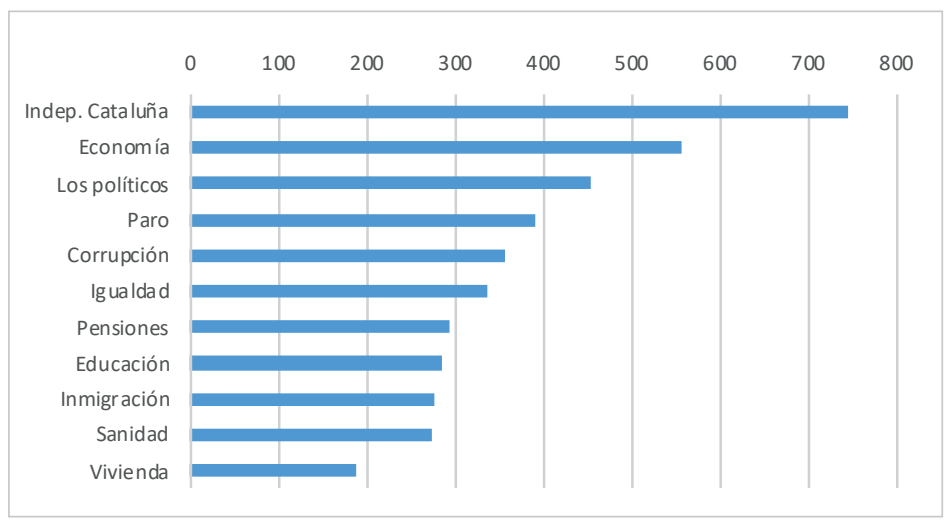

Gráfico 5. Temas tratados en los dos debates de la campaña en segundos (abril 2019)

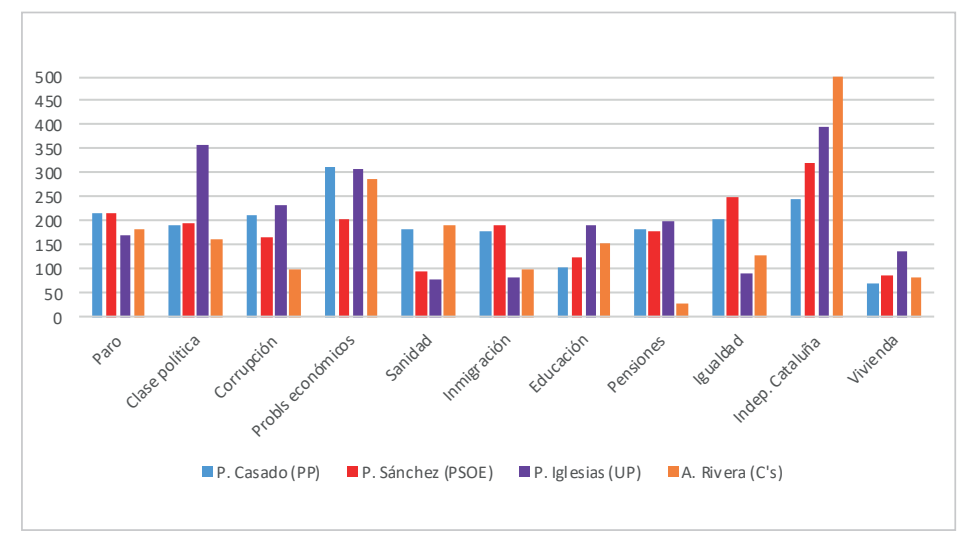

Gráfico 6. Temas tratados por cada candidato en los dos debates de la campaña en segundos (abril 2019) 
trata de un tema residual en su programa electoral y en sus interacciones en Twitter. Es muy probable que UP, igual que le sucede en los debates al PP con el tema corrupción, haya tenido que tratar la cuestión sin demasiada convicción y estableciendo puentes hacia otras ideas más favorecedoras como la concordia o la necesidad de negociación.

PSOE y PP también tratan el tema catalán, como no podría ser de otra forma al tratarse del tema estrella de los debates, aunque parecen más cómodos en otros lugares como la igualdad y el paro, en el caso del PSOE, o en economía, del lado del PP. UP, además de Cataluña, centra la mayor parte de sus esfuerzos en cuestiones como los políticos o la economía. Ciudadanos hace lo propio con un conglomerado de cuestiones económicas: economía, sanidad y paro.

\section{Conclusiones}

Los datos que hemos presentado aquí sobre programas electorales, visibilidad de temas en Twitter y debates nos han servido para abordar dos cuestiones diferentes, aunque complementarias. Por un lado, para ensayar una metodología complementaria e inclusiva que sea capaz

\section{La visibilidad de temas en Twitter se reali- za mucho más en las cuentas oficiales de los partidos que en las de los candidatos} de cuantificar los contenidos de la más escurridiza de todas las agendas, la agenda política. Por otro lado, esta investigación ha arrojado algo más de luz sobre la tematización que partidos y candidatos llevaron a cabo durante la campaña electoral de abril de 2019 en España. Un período convulso y trepidante en el que en una misma legislatura (2016-2019) han convivido dos presidentes de gobierno (Mariano Rajoy y Pedro Sánchez) y dos ejes temáticos muy diferentes: regeneración democrática/corrupción vs. independencia de Cataluña.

Si el análisis de contenido ad hoc y los barómetros mensuales del CIS nos ofrecen respectivamente las agendas mediática y pública, la cuestión se complica sobremanera cuando pretendemos conocer la agenda política. ¿Cuál es su composición aproximada para un período determinado?

Nuestra propuesta metodológica incluye una respuesta a este interrogante. Nos parece conveniente analizar la composición de la agenda política mediante dos movimientos. El primero se dirige hacia lo que hemos convenido en denominar aquí comunicación política "pura" y el segundo hacia otro tipo de repertorios que incluyen la influencia de otros partidos y candidatos.

Dentro de la comunicación política "pura" hemos incluido dos fuentes:

- programas electorales, y

- visibilidad temática en Twitter.

Los programas pueden ser considerados como una fuente más estática y conectada con cuestiones como la ideología y cleavages (fracturas sociales) como la clase social o la identidad nacional. Twitter en cambio, tiene la ventaja de conectar estas preferencias partidistas con la actualidad política del momento electoral, en nuestro caso, la campaña electoral de abril de 2019.

Pero nuestra propuesta de reconstrucción de la agenda política no termina aquí. A los programas y Twitter añadimos también los temas de debate público procedentes de los debates electorales. En nuestro caso de estudio se celebraron dos, uno en TVE (22 de abril) y otro en Atresmedia (23 de abril de 2019). Dando cabida a estos eventos introducimos en la agenda política la interacción con los demás actores del sistema político. Y lo hacemos precisamente para captar el componente sistémico y relacional que todo debate político contiene.

Buena parte de la tematización de partidos y candidatos depende de su interacción en el seno de una campaña con otros actores. Además, en los debates se introducen una serie de bloques temáticos que fuerzan a los candidatos a tomar posiciones en temas de campaña que en principio no formaban parte de su línea estratégica preferente.

Si aplicamos nuestro análisis a tres bandas (programas, Twitter y debates) para conocer la agenda política de la campaña electoral de abril de 2019, encontramos una agenda compuesta por tres temas principales: economía, los políticos y la independencia de Cataluña.

Los problemas de índole económica se encuentran tanto en los programas (rango 1) como en Twitter (rango 3) y debates (rango 2), además de en la agenda pública de los ciudadanos. Si sumamos esta cuestión con el paro (el CIS las desagrega) podemos situarlas, con mucha diferencia, en el rango 1 de importancia.

La regeneración democrática se encuentra también presente en la agenda política en un lugar muy relevante mediante el tema de debate "los políticos" que,

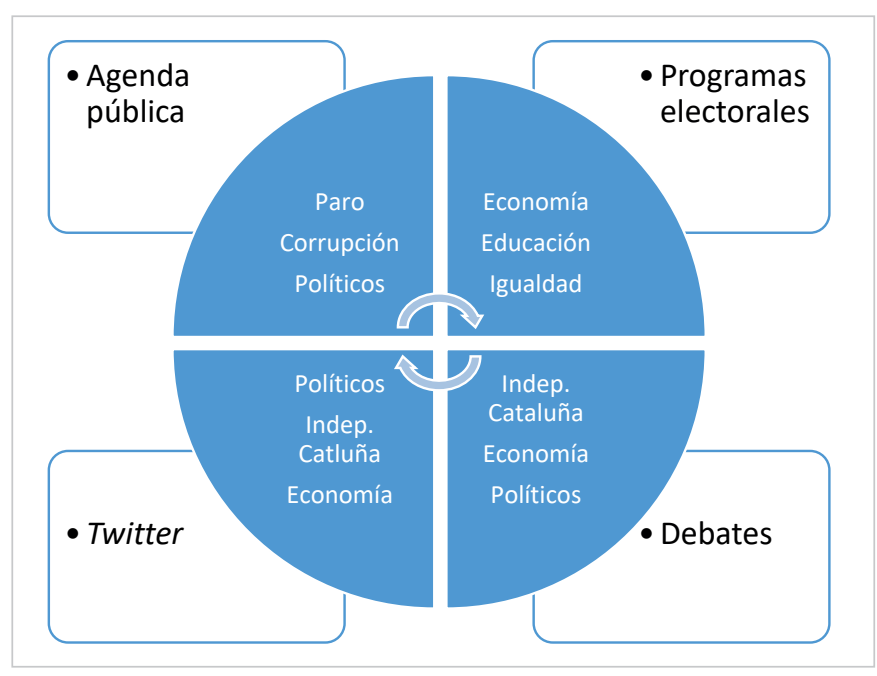

Gráfico 7. Agenda política en las elecciones generales de abril de 2019 
siguiendo la nomenclatura empleada por el CIS, se refiere a la percepción de la política como un problema para gestionar la sociedad. No cabe duda de que esta percepción crítica puede ser considerada como una dimensión importante de la denominada desafección política.
Los programas electorales tratan temas que enlazan con la ideología y las cosmovisiones más profundas de los partidos

La visibilidad de temas en Twitter y los debates electorales comparten su composición temática, pero no el orden. Es decir, se trata más de una cuestión de énfasis y jerarquía. Si en Twitter las cuestiones más importantes son los políticos, la independencia de Cataluña y la economía, en los debates electorales de campaña la prioridad corresponde a la independencia de Cataluña, la economía y los políticos.

Si analizamos las agendas políticas de los cinco principales partidos en las elecciones generales de abril de 2019 podemos ver algunas tendencias muy interesantes. En general, las formaciones políticas suelen construir sus programas electorales de forma muy similar a la agenda pública, es decir, a las preocupaciones de los ciudadanos recogidas mes a mes en los barómetros del CIS. Las cuestiones económicas y de servicios del estado del bienestar suelen ser las mayoritarias. Quizá las únicas excepciones a esta tendencia las constituyan Ciudadanos y su predilección por la corrupción y Vox y su relación conflictiva con el fenómeno migratorio.

Tabla 1. Agenda política de los partidos en las elecciones generales de abril de 2019

\begin{tabular}{|c|c|c|c|c|c|}
\hline & PSOE & $P P$ & C's & UP & Vox \\
\hline Programas & $\begin{array}{c}\text { Economía } \\
\text { Educación } \\
\text { Paro }\end{array}$ & $\begin{array}{l}\text { Economía } \\
\text { Educación } \\
\text { Igualdad }\end{array}$ & $\begin{array}{l}\text { Corrupción } \\
\text { Economía } \\
\text { Educación }\end{array}$ & $\begin{array}{l}\text { Economía } \\
\text { Paro } \\
\text { Igualdad }\end{array}$ & $\begin{array}{l}\text { Inmigración } \\
\text { Economía } \\
\text { Educación }\end{array}$ \\
\hline Twitter & $\begin{array}{c}\text { Los políticos } \\
\text { Indep. Cataluña } \\
\text { Paro }\end{array}$ & $\begin{array}{c}\text { Los políticos } \\
\text { Paro } \\
\text { Indep. Cataluña }\end{array}$ & $\begin{array}{c}\text { Indep. Cataluña } \\
\text { Economía } \\
\text { Los políticos }\end{array}$ & $\begin{array}{l}\text { Los políticos } \\
\text { Paro } \\
\text { Economía }\end{array}$ & $\begin{array}{c}\text { Los políticos } \\
\text { Indep. Cataluña } \\
\text { Economía }\end{array}$ \\
\hline Debates & $\begin{array}{l}\text { Indep. Cataluña } \\
\text { Igualdad } \\
\text { Paro }\end{array}$ & $\begin{array}{c}\text { Economía } \\
\text { Indep. Cataluña } \\
\text { Paro }\end{array}$ & $\begin{array}{l}\text { Indep. Cataluña } \\
\text { Economía } \\
\text { Sanidad }\end{array}$ & $\begin{array}{l}\text { Indep. Cataluña } \\
\text { Los políticos } \\
\text { Economía }\end{array}$ & No participó \\
\hline
\end{tabular}

En Twitter la agenda política adquiere un tono más crítico, de mayor confrontación para con los demás contendientes de la campaña a través del tema de debate "los políticos". La independencia de Cataluña es otro tema estrella en Twitter seguida de las cuestiones económicas (paro y economía).

Por último, hay que destacar que los debates se parecen mucho más a Twitter que a la composición temática de los programas electorales. La independencia de Cataluña es ahora el tema más tratado, junto a los temas de debate de corte económico.

En resumen, nuestra propuesta para aproximarnos a la agenda más complicada de todas tiene en cuenta dos movimientos complementarios. Por un lado la comunicación política "pura" (programas electorales y visibilidad en Twitter) y por otro aquella otra que toma en consideración la relación con los demás partidos y candidatos del sistema.

Pero se trata sólo de una aproximación, ya que la composición exacta de los componentes temáticos de la agenda de nuestros representantes resulta imposible o, cuanto menos, mucho más complicada que en el caso de la agenda mediática y las agendas ciudadanas. En definitiva, cuando trabajamos con la agenda política debemos aprender a vivir con un cierto grado de incertidumbre. Esperamos que esta investigación habrá servido para reducirla.

\section{Notas}

1. Este caso hace referencia a una red de corrupción política vinculada al Partido Popular cuya investigación se inició en 2007 en la Fiscalía Anticorrupción y que más tarde sería asumida por la Audiencia Nacional.

2. EI CIS codifica la percepción ciudadana hacia la clase política como un problema para la sociedad con el siguiente literal: "Los/as políticos/as en general, los partidos y la política".

3. El referéndum se celebró el 1 de octubre de 2017 y se basó en una ley aprobada por el Parlament de Catalunya el 6 de septiembre, considerada ilegal por ir en contra del Estatuto de Autonomía de Cataluña. Esta ley fue suspendida cautelarmente el día anterior a su publicación por el Tribunal Constitucional.

4. Este artículo de la Constitución Española permite al gobierno central hacerse con el control de una Comunidad Autónoma si se entiende que ésta atenta contra el interés general de España. La aplicación de este articulo supuso la destitución del Govern catalán en pleno y la convocatoria de nuevas elecciones.

5. Sobre este tema de debate Ciudadanos ya había conseguido unos excelentes resultados en las elecciones autonómicas catalanas de 2017, donde fue la opción política más votada: 36 diputados con el 25,35\% del voto.

http://gencat.cat/economia/resultats-parlament2017/09AU/DAU09999CM.htm?lang=es 
6. https://elpais.com/especiales/2019/elecciones-generales/programas-electorales La aplicación permite también la descarga de los programas íntegros en formato PDF.

7. El 16 de abril la JEC prohíbe a Vox asistir al debate "a cinco" por no disponer, en aquellos momentos, de representación parlamentaria.

8. La pregunta de agenda pública y personal suele insertarse al principio de cada barómetro y tiene una formulación muy similar.

Agenda pública: “¿Cuál es, a su juicio, el principal problema que existe actualmente en España? ¿Y el segundo? ¿Y el tercero?”.

Agenda personal: “¿Y cuál es el problema que, a Ud., personalmente, le afecta más? ¿Y el segundo? ¿Y el tercero?”.

9. El promedio se ha calculado entre julio de 2016 y febrero de 2019, es decir, se han tenido en cuenta 29 barómetros. Se trata de una media simple.

10. Por esta circunstancia estos tres temas no han sido tenidos en cuenta desde el principio de la legislatura (barómetro de julio 2016) sino desde el momento en el que toman importancia. En el caso del Procés catalán, el barómetro de octubre de 2017; para las pensiones, el de enero de 2018 y finalmente, se comienza a tener en cuenta la inmigración desde el estudio del CIS de abril de 2018.

11. En marzo de 2018 los colectivos de los pensionistas se manifestaron en las principales ciudades españolas para exigir unas pensiones dignas. Esta circunstancia introdujo el tema en el debate público, tal y como los datos de agenda pública del CIS indican.

\section{Bibliografía}

Benedicto, Jorge (1995). “La construcción de los universos políticos de los ciudadanos”. En: Benedicto, Jorge; Morán-Calvo, María-Luz (eds.). Sociedad y política. Temas de sociología política. Madrid: Alianza Editorial, pp. 227-268. ISBN: 84 20681520

Carballo, Marita; López-Escobar, Esteban; McCombs, Maxwell E. (2018). “Communication, public opinion, and democracy: New challenges". Communication \& society, v. 31, n. 4, pp. 121-134.

https://revistas.unav.edu/index.php/communication-and-society/article/view/35676

Castromil, Antón R. (2017). Ciencia política para periodistas. Ideas para una información más rigurosa. Barcelona: Editorial UOC. ISBN: 9788491167549

Castromil, Antón R.; Rodríguez-Díaz, Raquel (2019). “Del cara a cara a los debates a cuatro en España”. En: Conde-Vázquez, Erica; Fontenla-Pedreira, Julia; Rúas, José. Debates electorales televisados: del antes al después. La Laguna: Cuadernos Artesanos de Comunicación, n. 154, pp. 27-47. ISBN: 9788417314149

https://www.ucm.es/data/cont/media/www/pag-79285/castromil_y_rodriguez.pdf

Chavero-Ramírez, Palmira; González, Juan-Jesús; Bouza, Fermín; Castromil, Antón; Rodríguez-Díaz, Raquel (2013). “La mediatización de la agenda política: la discusión del pacto social como conflicto de agendas, 2009-2011". Revista latina de comunicación social, n. 68, pp. 639-655.

http://www.revistalatinacs.org/068/paper/994_Complutense/RLCS_paper994.pdf

Gallego-Reguera, María; Martínez-Martínez, Isabel (2019). “iOrganización! Producción y realización de los debates electorales en España (1993-2016)”. En: Conde-Vázquez, Erica; Fontenla-Pedreira, Julia; Rúas, José. Debates electorales televisados: del antes al después. La Laguna: Cuadernos Artesanos de Comunicación, n. 154. ISBN: 9788417314149 https://dialnet.unirioja.es/servlet/articulo?codigo=6995381

García-Marín, Javier; Calatrava, Adolfo; Luengo, Óscar G. (2018). “Debates electorales y conflicto. Un análisis con máquinas de soporte virtual (SVM) de la cobertura mediática de los debates en España desde 2008". El profesional de la información, v. 27, n. 3, pp. 624-632.

https://doi.org/10.3145/epi.2018.may.15

Guillen, Alberto; Rodríguez-Díaz, Raquel (2017). “Frames y agendas durante el proceso soberanista catalán (20132015)". Sur le journalisme, About journalism, Sobre jornalismo, v. 6, n. 2, pp. 140-157.

http://www.surlejournalisme.kinghost.net/rev/index.php/slj/article/view/321

Guo, Lei; McCombs, Maxwell (eds.) (2015). The power of information networks: New directions for agenda setting. New York. Routledge. ISBN: 9781138847750

Hallin, Daniel C.; Mancini, Paolo (2004). Comparing media systems. Three models of media and politics. New York: Cambridge University Press. ISBN: 9780521543088 
Kim, Yeojin; Kim, Youngju; Zhou, Shuhua (2017). "Theoretical and methodological trends of agenda-setting theory. A thematic analysis of the last four decades of research". The agenda setting journal, v. 1, n. 1, pp. 5-22.

https://doi.org/10.1075/asj.1.1.03kim

Lippmann, Walter (2011). El público fantasma. Madrid: Genueve Ediciones. ISBN: 9788493855703

Lipset, Seymour M.; Rokkan, Stein (2001). "Estructuras de división, sistemas de partidos y alineamientos electorales". En: Batlle-Rubio, Albert (ed.). Diez textos básicos de ciencia política. Barcelona: Ariel, pp. 231-273. ISBN: 8434416859

López-López, Paulo-Carlos; Vásquez-González, Javier (2018). “Agenda temática y Twitter: elecciones presidenciales en América Latina durante el período 2015-2017". El profesional de la información, v. 27, n. 6, pp. 1204-1214.

https://doi.org/10.3145/epi.2018.nov.04

Mazzoleni, Gianpietro (2010). La comunicación política. Madrid: Alianza Editorial. ISBN: 9788420669403

McCombs, Maxwell E. (2006). Estableciendo la agenda. El impacto de los medios en la opinión pública y en el conocimiento. Barcelona: Paidós Comunicación. ISBN: 8449318696

McCombs, Maxwell E.; Shaw, Donald L. (1972). "The agenda-setting function of mass media”. The public opinion quarterly, v. 36, n. 2, pp. 176-187.

https://www.jstor.org/stable/2747787

McCombs, Maxwell E.; Shaw, Donald L.; Weaver, David H. (2014). "New directions in agenda-setting theory and research". Mass communication and society, v. 17, n. 6, pp. 781-802.

https://doi.org/10.1080/15205436.2014.964871

Miralles, Ana-María (2011). El miedo al disenso. El disenso periodístico como expresión democrática de las diferencias y no como provocación de violencia. Barcelona: Gedisa. ISBN: 9788497845748

Navarro-Sorolla, María; Rodríguez-Díaz, Raquel (2017). “Política mediatizada: El caso español durante el periodo 20082012". Index. comunicación: Revista científica de comunicación aplicada, v. 7, n. 1, pp. 143-163.

http://journals.sfu.ca/indexcomunicacion/index.php/indexcomunicacion/article/view/315/288

Ortega, Félix (2011). La política mediatizada. Madrid: Alianza Editorial. ISBN: 9788420650654

Restrepo-Echavarría, Néstor-Julián; Rodríguez-Díaz, Raquel; Castromil, Antón R. (2018). "Proposal of an indicator to measure the professionalization of election campaigns: The case of Colombia". El profesional de la información, v. 27, n. 2, pp. 289-299.

https://doi.org/10.3145/epi.2018.mar.07

Rodríguez-Díaz, Raquel (2004). Teoría de la agenda-setting. Aplicación a la enseñanza universitaria. Alicante: Obets. ISBN: 8460924602

http://rua.ua.es/dspace/handle/10045/2297

Ruiz-del-Olmo, Francisco-Javier; Bustos-Díaz, Javier (2017). "La evolución del debate televisivo como herramienta de comunicación política. Análisis del caso español: de la televisión a Twitter". Informação \& sociedade, v. 27, n. 2, pp. $235-252$. http://www.periodicos.ufpb.br/ojs/index.php/ies/article/view/32491

Sánchez-Aranda, José-Javier (2005). "Análisis de contenido cuantitativo de medios". En: Berganza-Conde, Rosa; RuizSan-Román, José-Antonio (coords.). Investigar en comunicación. Guía práctica de métodos y técnicas de investigación social en comunicación. Madrid: McGraw-Hill. ISBN: 8448198255

Snow, David (2004). "Framing processes, ideology, and discursive fields". En: Snow, David A.; Soule, Sarah A.; Kriesi, Hanspeter. The Blackwell companion to social movements. Malden: Blackwell Publishing Ltd. ISBN: 9780631226697 https://doi.org/10.1002/9780470999103.ch17

Snow, David; Benford, Robert (2006). "Marcos maestros y ciclos de protesta". En: Chihu, Aquiles. El análisis de los marcos en la sociología de los movimientos sociales. Ciudad de México: Editorial UAM. ISBN: 9707017082

Strömbäck, Jesper (2008). "Four phases of mediatization: An analysis of the mediatization of politics". The international journal of press/politics, v. 13, n. 3, pp. 228-246.

https://doi.org/10.1177/1940161208319097

Vu, Hong-Tien; Guo, Lei; McCombs, Maxwell E. (2014). “Exploring 'the world outside and the pictures in our heads': A network agenda-setting study". Journalism \& mass communication quarterly, v. 91, n. 4, pp. 669-686.

https://doi.org/10.1177/1077699014550090

Wagner, Markus (2016). "Selective exposure, information utility, and the decision to watch televised debates". International journal of public opinion research, v. 29, n. 4, pp. 533-553.

https://doi.org/10.1093/ijpor/edw016 


\section{Anexos}

Gráfico A1: Temática de las interacciones en Twitter de los partidos (abril de 2019)
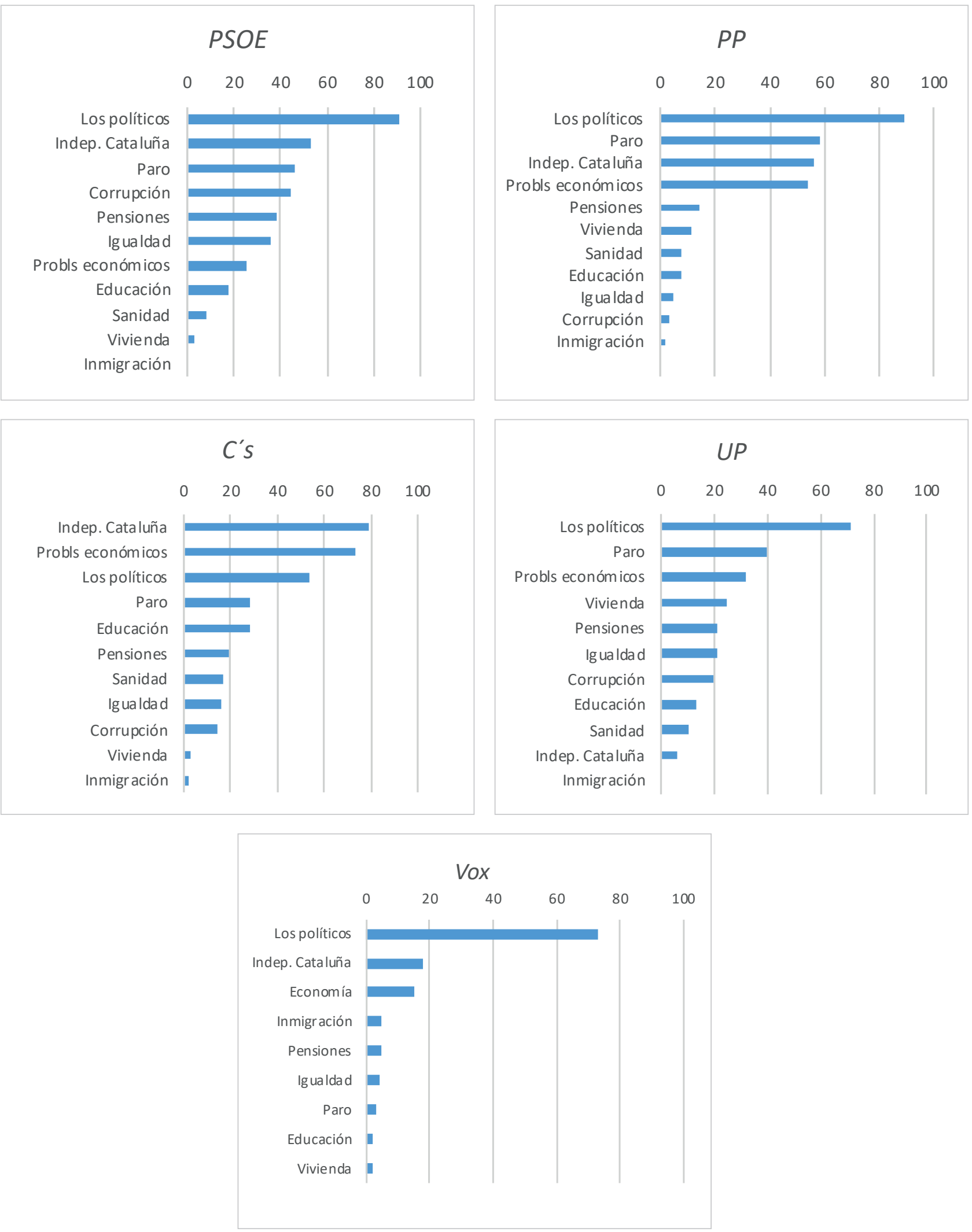